\title{
Neu: 300.000 Bände frei zugänglich in sachlicher Ordnung Ein Praxisbericht
}

Die Württembergische Landesbibliothek hatte sich im Laufe ihrer 255-jährigen Geschichte zu einer klassischen Magazinbibliothek entwickelt. Im Sommer 2020 standen $97 \%$ der insgesamt über 6 Millionen Medieneinheiten auf 13.000 qm (96.000 Regalmeter) in geschlossenen Büchermagazinen in den zwei Untergeschossen der Bibliothek sowie in einem Außenmagazin außerhalb der Stadtgrenzen Stuttgarts in Fellbach mit weiteren 3.300 qm Fläche (10.500 Regalmeter). Mit Bezug des Erweiterungsbaus bot sich erstmals die Möglichkeit, den Nutzern der Bibliothek einen umfangreichen Freihandbestand anzubieten.

Die ursprünglichen Planungen sahen ein rund 500.000 Bände umfassendes Freihandmagazin an der Konrad-Adenauer-Straße vor, aus dem der Nutzer Bände direkt entnehmen und über Selbstverbuchung hätte ausleihen können. ${ }^{1}$

Ihre Aufstellung sollte platzsparend nach fortlaufender Magazinsignatur (Numerus Currens) erfolgen; zudem wollte man durch diese Form der Aufstellung eine aufwendige Neuetikettierung und ein anspruchsvolles Umsortieren umgehen.

Als sich jedoch abzeichnete, dass die Sanierung des Bestandsgebäudes (voraussichtlich ab 2021) einen Komplettauszug voraussetzte, musste auch die Bespielung des Erweiterungsbaus neu bedacht werden. Rund 70.000 Bände Präsenzbestand aus Haupt- und z. T. auch Sonderlesesaal benötigten einen neuen Aufstellungsort im Erweiterungsbau. Diese Bände, die zunächst für das 4. OG im Erweiterungsbau vorgesehen wurden, waren seit 2018 auf das Signaturensystem der Regensburger Verbundklassifikation (RVK) umgearbeitet worden. Die RVK ist eine in 34 Fachsystematiken unterteilte Universalklassifikation, die von 150 wissenschaftlichen Bibliotheken in Deutschland und im deutschsprachigen Raum zur Systematisierung der Literatur genutzt wird.

Sie verfügt über eine Million Systemstellen, denen Bücher zugeordnet werden können, z. B.

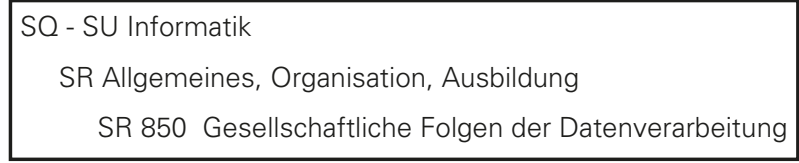

oder

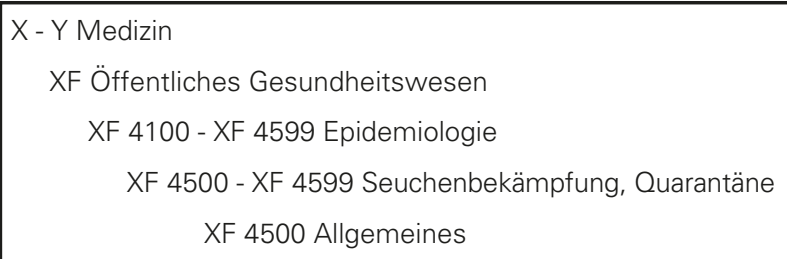

Als Aufstellungssystematik erlaubt die RVK dem Nutzer eine thematische Sichtung am Regal. Auf Initiative von Dr. Rupert Schaab und mit breiter Zustimmung aus dem Kollegium fiel im Oktober 2019 die Entscheidung, auch den ausleihbaren Freihandbestand der WLB künftig nach der Regensburger Verbundklassifikation aufzustellen. Ziel sollte ein systematisch nach Fächern aufgestellter Medienbestand sein, bei dem ausleihbare und nicht-ausleihbare Bände ineinander sortiert an gleicher inhaltlicher Stelle stehen. Bibliotheksnutzer finden Titel aus ihrem Fachgebiet über den Katalog in räumlich festgelegten Regalreihen und können daneben weitere Bücher desselben Interessengebiets finden. Eine systematische Freihandaufstellung fördert die Sozialisation von Nutzern der gleichen Disziplinen und sie trägt dazu bei, das Wissenschaftssystem als Raum erfahrbar zu machen. Für die innerbibliothekarischen Prozesse stellt sie allerdings einen Paradigmenwechsel dar, der mit einem erheblichen Mehraufwand, sowohl für die rückwirkend umgestellten Medien als auch für den laufenden Neuzugang einhergeht.

\section{Automatische Datenanreicherung}

Die Zeit war knapp: Von den ersten Ideen im Oktober 2019 bis zur Inbetriebnahme des Erweiterungsbaus im Sommer 2020 blieben nur wenige Monate und bis dahin sollten rund 250.000 Bände nach dem neuen Signaturensystem bereitstehen. Eine intellektuelle Vergabe von Systemstellen, 
selbst unter Hinzuziehung von Fremddaten, wie dies beim Lesesaalbestand gemacht wurde, war von Anfang an ausgeschlossen. Ein automatisiertes, auf Fremddatenübernahme basierendes Verfahren musste her. Doch dabei stellte sich das Problem der grundsätzlichen Heterogenität von RVK-Signaturen: Die RVK sieht in ihrer klassischen Ausprägung eine Individualisierung der Signatur über eine Vergabe einer Cutter-Sanborn-Notation (CSN) vor, die an die systematische Notation angehängt wird, z. B.

\begin{tabular}{|l|l|l|l|}
\hline PO 3555 & J64 & D6 & $\mathbf{- 2}$ \\
\hline $\begin{array}{l}\text { RVK- } \\
\text { Notation }\end{array}$ & CSN & ggf. 2. CSN & ggf. Bandzählung \\
\hline
\end{tabular}

Die Vergabe der CSN erfolgt zwar nach einer festgelegten Reihenfolge von Ordnungsbegriffen (Autor, Herausgeber, beteiligte Person, Ordnungswort aus dem Titel), darüber hinaus ist sie jedoch nicht normiert und jeweils abhängig vom tatsächlich vor Ort vorhandenen Literaturbestand. Eine Übernahme von RVK-Signaturen mit CutterSanborn-Notationen hätte komplizierte Verfahren der Dublettenprüfung vorausgesetzt, die so nicht zu leisten gewesen wären. Aus pragmatischen Gründen entschied man sich deshalb gegen eine Vercutterung und für eine Individualisierung anhand der WLB-Magazinsignatur, die nach dem Numerus-currens-Prinzip aufgebaut ist.

Das Numerus-currens-Signaturensystem der WLB wurde im Jahr 1950 mit dem Jahresring 1 begonnen. An den Jahresring fügt sich hinter dem Querbalken eine laufende Nummer an. Formatangaben werden durch einen an den Jahresring angehängten Buchstaben ausgedrückt und nicht ausleihbare Bestände stehen auf einem speziellen Nummernkontingent (40.000 ff). Es handelt sich um Individualsignaturen mit einer chronologischen Ordnung, die in Teilen den Zugang der Bücher in die Bibliothek abbildet.

Ein Beispiel: Der Band mit der Signatur 40a/2227 kam im Zugangsjahr 1990 (Ring 40) als 2227-ter Band in die Bibliothek und ist von seinen Maßen her ein Quartband (,a").

Für den ausleihbaren Freihandbereich wurde also das neue Signaturensystem Notation + Magazinsignatur (Numerus currens) eingeführt. Darüber hinaus wurde beschlossen, dass nur diejenigen Medien in den Freihandbereich aufge-

\begin{tabular}{l|l}
\hline SR 850 & $69 / 17210$ \\
RVK-Notation & Magazinsignatur (ggf. Bandzählung)
\end{tabular}

stellt werden, bei denen Notationen aus anderen Bibliotheken übernommen werden können. Eine intellektuelle/manuelle Umarbeitung von Bänden wurde mit Ausnahme der im Bereich NZ 70000 Landesgeschichte Baden-Württemberg aufzustellenden Literatur von vornherein ausgeschlossen.

Die automatische Datenanreicherung wurde durch Mitarbeiter des Bibliotheksservice-Zentrums (BSZ) nach umfangreichen Vorgaben der WLB programmiert und umgesetzt. Berücksichtigt wurden nur Medien, die ausleihbar sind; ausgeschlossen wurden u. a. Kleinschriftmaterial (Kapselschriften), Folio-Bände und Zeitschriften. Bände, die künftig im Bereich NZ 70000 Landesgeschichte BadenWürttemberg stehen sollen, wurden ebenfalls ausgeschlossen, denn dieser Systematikbereich wurde erst Ende 2019 neu in die RVK eingebracht, Fremddaten sind daher nicht vorhanden und die Notationen müssen allesamt intellektuell durch Mitarbeiter vergeben werden (vgl. nachfolgender Artikel).

\section{Verwendung von Daten aus Culturegraph- Projekt}

Datengrundlage waren die im gemeinsamen Bibliothekskatalog K10plus in Kategorie 5090 im Titeldatenbereich vorhandenen und mit der RVKNormdatei verknüpften Notationen. Dank der zahlreichen RVK-Anwender aus zehn Bundesländern, die sich an diesem Verbundkatalog beteiligen, aber auch dank regelmäßiger Fremddateneinspielungen durch das BSZ war die Datenlage sehr günstig. Durch Beschränkung auf diese in der gemeinsamen Katalogisierungsdatenbank bereits erfassten Titel konnte man auf zusätzliche Verfahren wie Titel- oder ISBN-Abgleich verzichten. Verwendet wurde jeweils die Notation, die dem Fachgebiet, für das das Buch ursprünglich gekauft wurde, am nächsten kam. Die im Exemplarsatz in Feld 8520 verzeichnete Etat-Fachgruppe wurde dazu in einer Konkordanz auf die jeweilige RVK-Fachsystematik abgebildet. Durch Ausschluss bzw. nachrangige Gewichtung bestimmter Notationen und Notationsbereiche gelang es, Doppelstellen gezielt zu sperren und Einfluss auf die Vergabe von Signatu- 


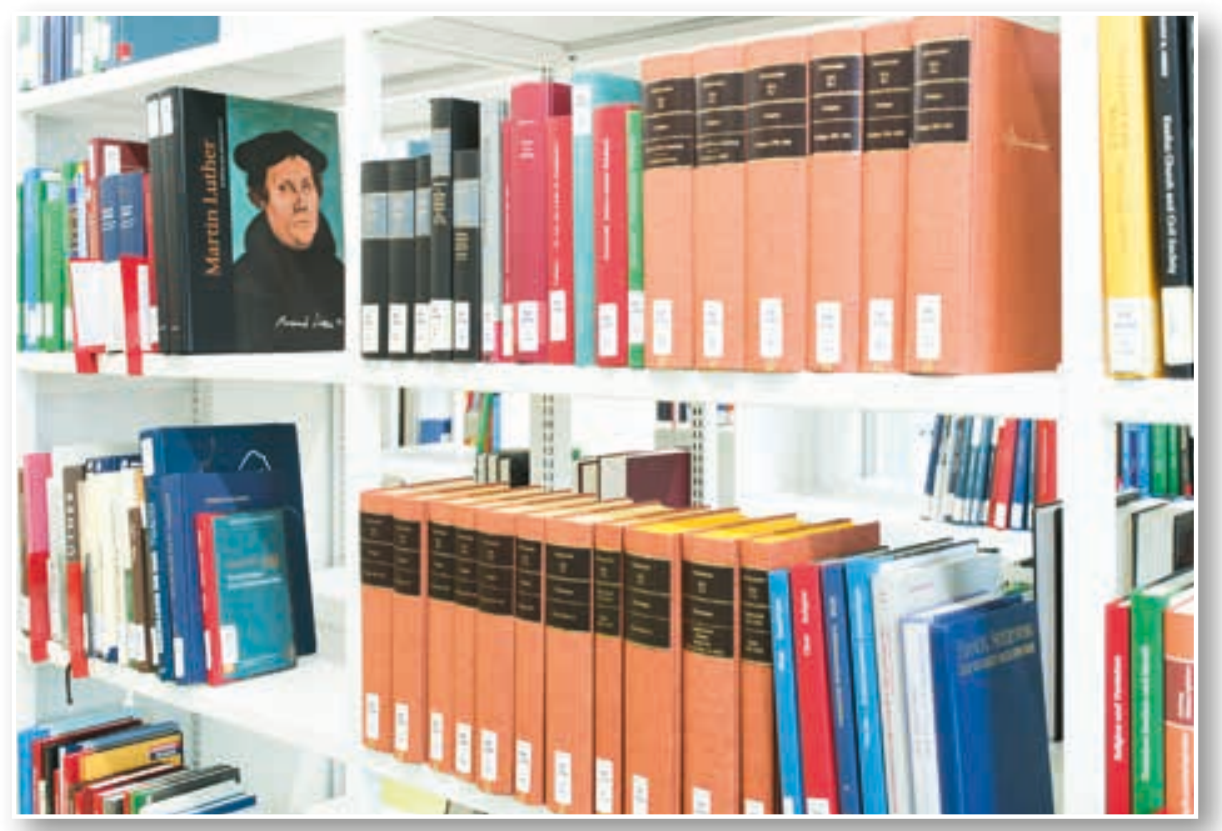

die in der WLB zu einem einzigen Band zusammengebunden wurden). Hier und in anderen Fällen war eine Datenbereinigung durch Mitarbeiterinnen der Medienbearbeitung und der Leihstelle nötig. Die durch die IT-Abteilung ermittelten aDIS-Exemplare wurden in aufbereiteter Form (interne Datensatznummer, neue Signatur) der Firma aStec zur Verfügung gestellt. Neben der Eintragung der Signatur wurden dort automatisiert weitere Felder geändert: der Standort, der Medientypcode zur Steuerung der Ausleihberechtigung und die Bestellbarkeit. Exemplare, die bei dieAbb. 1: Luther in seinem Umfeld

ren und somit die künftige Aufstellung der Medien zu nehmen. Bei mehrbändigen Werken wurde versucht, allen Einzelbänden dieselbe Notation zu geben, damit die Bände zusammenstehen.

Wo im K10plus-Katalog keine Notation ermittelt werden konnte, wurde der von der DNB entwickelte Culturegraph-Dienst herangezogen, welcher die Metadaten der deutschen und österreichischen Bibliotheksverbünde sowie der DNB vernetzt zur Verfügung stellt. Mit diesem auf einem Algorithmus basierenden und mit Werkclustern operierenden Programm wurden weitere 5 bis 10\% der Titel mit Notationen versehen. Von den aus formalen Gründen theoretisch in Frage kommenden 245.000 Bänden der ersten Tranche, die die neuesten 10 Jahresringe (Zugangsjahre 20102019) umfassten, wurden mittels beider Verfahren insgesamt 160.000 Bände (65\%) mit Notationen angereichert und im Datensatz des Exemplars im K10plus mit der neuen Signatur versehen.

\section{Zuordnung zu Exemplaren im Lokalsystem}

Aufgrund der getrennten Datenhaltung von einerseits Titeldaten im K10plus und andererseits Lokalund Ausleihdaten im Lokalsystem aDIS/BMS musste in einem nächsten Schritt ein Titelabgleich zwischen K10plus- und aDIS-Exemplarsatz erfolgen. Zum Abgleich wurde die Magazin-Signatur herangezogen. Manche Exemplare haben in aDIS und K10plus leicht unterschiedliche Signaturen (z. B. zwei Bände eines mehrbändigen Werks, sem Verfahren nicht zugeordnet werden konnten, insgesamt 142 Exemplare in der ersten Tranche, wurden dem Ansprechpartner im BSZ zugesandt, der die Signaturerstellung wieder rückgängig machte. Diese wenigen Exemplare verblieben im Magazin.

\section{Etikettendruck}

Anschließend wurden die Signaturen der ermittelten Exemplare durch die IT-Abteilung für den Etikettendruck aufbereitet: Im Hinblick auf die z. T. erheblich differierenden Breiten bei Buchrücken wurden pro Signatur drei unterschiedlich große Etiketten erzeugt. Jedes dieser drei Etiketten hat einen unterschiedlichen

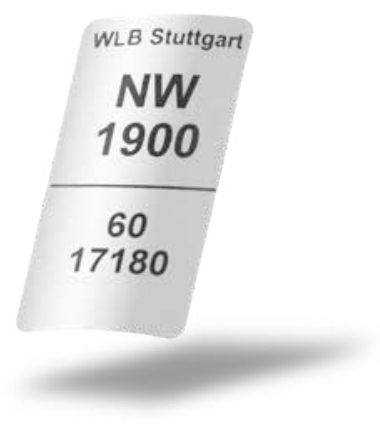
Aufbau: Beim großen Etikett wird die Signatur in großer Schrift auf fünf Zeilen aufgedruckt, beim kleinsten in Querformat auf nur noch einer Zeile und unter Auslassung von Bandangaben. Der Zeilenumbruch ist dabei genau definiert und so kompliziert, dass diese Arbeit nicht an einen Dienstleister gegeben werden konnte.

Im Anschluss mussten die Signaturen so sortiert werden, dass die ausgedruckten Signaturbögen nach der Laufrichtung am Regal angeordnet sind. Das erledigte eine zu programmierende Spezialsortierung nach dem Altsignaturanteil (69/17210) einer Signatur (SR 850 69/17210), also einer 
Sortierung nach dem hinteren Signaturteil unter Auslassung der Notation. Die Etiketten wurden schließlich durch die Firma Pleuser auf handliche DIN-A4-Bögen ausgedruckt.

Die WLB verfügt bereits seit einigen Jahren über ein eigenentwickeltes Etiketten-Druckprogramm für Einzelsignaturen, das für den Neuzugang der RVK-Präsenzbestände verwendet wird. Dabei liest der Mitarbeiter der Ausstattung die RVK-Signatur über die Mediennummer im Buch ein und erhält die Etiketten in den drei genannten Größen mit passendem Zeilenumbruch. Diese Software musste ebenfalls für den laufenden Neuzugang der ausleihbaren Freihandbestände weiterentwickelt und auf das Signaturensystem Notation + Magazinsignatur angepasst werden.

\section{Von der Etikettierung zur Sortierung}

Nun konnte endlich mit der "praktischen" Arbeit begonnen werden. Für das Bekleben der Exemplare mit neuen Etiketten waren täglich von 8 bis 20 Uhr in 3 Schichten à 4 Stunden zeitgleich 3 Teams aus einem Mitarbeiter und einer studentischen Hilfskraft im Einsatz, dazu zeitweise ein Springer, insgesamt also 18 bis 21 Personen pro Tag. Dieser hohe Personaleinsatz beschleunigte die Umsetzung erheblich. So waren bereits nach 7 Wochen alle 158.000 Etiketten verklebt - wobei Etiketten für ausgeliehene Medien zurückgehalten und erst bei Rückgabe angebracht werden.

Die Sortierung der neuen Signaturen (z. B. „SR 850 69/17210“) erfolgte schrittweise nach einem kaskadierenden Verfahren - auch hier wiederum in Zweierteams: Ein Mitarbeiter zog nacheinander alle Medien mit einem bestimmten Anfangsbuchstaben, z. B. alle mit "S", aus dem Regal und stellte sie in ein fahrbares Bücherregal. Der Teamkollege nahm das Regal mit, konzentrierte sich ausschließlich auf den zweiten Buchstaben und zog nacheinander aus dem fahrbaren Regal zunächst alle Medien mit dem Zweitbuchstaben A und stellte sie ins "Zwischensortierregal", anschließend alle mit Zweitbuchstaben B, stellte sie mit Abstand ins Zwischensortierregal usw.; dann bekam er den zweiten Wagen von Erstbuchstabe "S" und begann wieder bei "A". Die anderen Teams machten das gleiche mit anderen Anfangsbuchstaben.

Nach Fertigstellung dieses ersten Sortierschritts (für den Buchstaben „S") begann der zweite
Sortierschritt: Jetzt waren die beiden folgenden Ziffern dran, die wiederum analog sortiert wurden, d. h. ein Teammitglied konzentrierte sich nur auf die dritte, das andere auf die vierte Stelle der Signatur. Diese Schritte wurden so lange wiederholt, bis der je nach Fachgebiet unterschiedlich lange Notationsteil (hier "SR 850“) vollständig sortiert war. Durch das durchgängige Bearbeiten der Bücher "von links nach rechts" und angesichts der Tatsache, dass der hintere Teil (hier "69/17210“) schon immer sortiert war, endete damit die Sortierung, wobei die einzelnen Bände zu jedem Zeitpunkt mit geringem Aufwand für die Ausleihe gefunden werden konnten. Diese Art der Sortierung konnte nur deshalb realisiert werden, weil durch den Erweiterungsbau ein zusätzlicher Magazinabschnitt mit einer Fläche von ca. 1.000 qm hinzukam. Dieser Abschnitt 8 befindet sich auf der Ebene des 1. UG und ist, über eine Rampe, mit dem bestehenden Tiefenmagazin verbunden. Dort konnten fast alle der 158.000 Bände vorsortiert werden, die dann, ab dem 15. Juli, in den Erweiterungsbau umgestellt wurden.

\section{Regalplanung für die endgültige Aufstellung}

Parallel zu diesen Arbeiten ging es für die Mitarbeiter des Magazins und des Lesesaals an die Planung, welche Bände in welchem Stockwerk aufgestellt werden können. Entschieden wurde, die Bände komplett nach der von der RVK vorgegebenen Ordnung aufzustellen, also mit "A" im ersten Stock zu beginnen bis zu "Z" im 4. Obergeschoss. Außerdem sollten die nicht ausleihbaren Bände des bisherigen Lesesaals pro Notation (z. B. "SR 850") in die ausleihbaren Bände einsortiert werden. Und nicht zuletzt sollte genügend Platz für die kommenden fünf bis sieben Jahre bleiben, um nicht vor dem Rückzug in den dann sanierten Altbau bereits erste Bände wieder ins Magazin zurückstellen zu müssen.

Dazu wurde das Rauminformationssystem Mapongo, das seit Langem ein 3D-Modell für das Bestandsgebäude enthält, um eine Komponente zur Regalplanung ergänzt. Es ermöglichte die Einspielung aller dort aufzustellenden Exemplare und führte nach vorheriger Auswahl des Fachgebiets und des Standorts eine teilautomatische Befüllung durch, inkl. Platz für Zeitschriftenboxen, ferner nicht ausleihbare und ausleihbare Medien sowie Reserve für Neuzugang. 


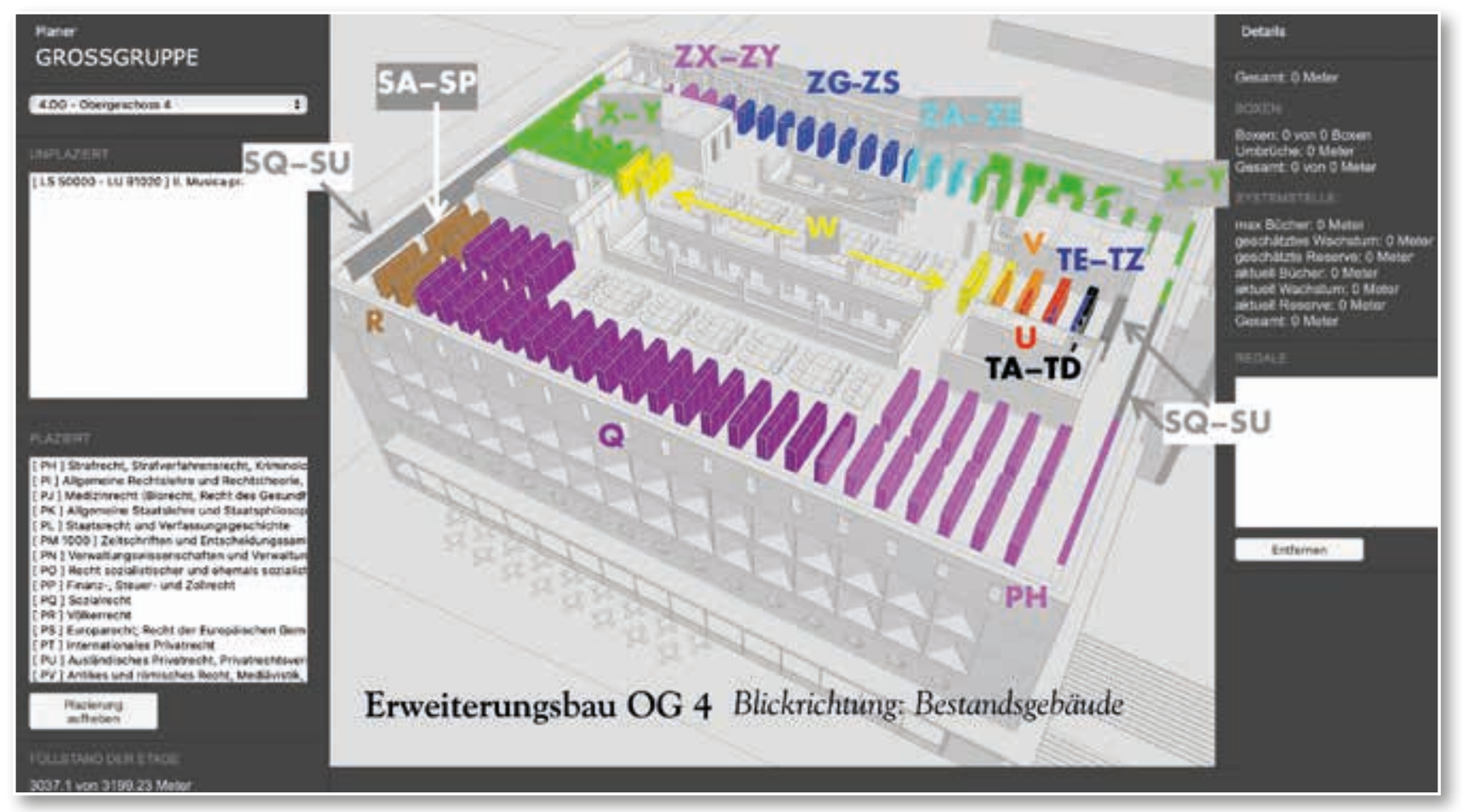

\section{Aufstellung und Systemanpassungen zur Inbetriebnahme}

Einige Wochen vor Inbetriebnahme des Erweiterungsbaus startete die tatsächliche Aufstellung im Gebäude: Aus Mapongo wurden Packlisten erzeugt, damit die Magazinmitarbeiter und die Umzugsfirma den genauen Zielstandort jedes Buch ablesen konnten. Zunächst wurden innerhalb von drei Wochen die Freihandbestände eingeräumt. In der Woche vor Eröffnung erfolgt die Verlagerung und Einsortierung der Lesesaalbände.

Parallel dazu starteten abschließende Tests und Arbeiten im Lokalsystem aDIS/BMS: Die Bände, die zur Eröffnung im Freihandbereich stehen, werden nicht mehr via Katalog bestellbar sein, denn die Nutzer können diese Bücher selbst am Regal entnehmen. Für die gebende Fernleihe, also Bestellungen von Nutzern fremder Bibliotheken, werden die Bücher aber weiterhin bestellbar sein. Außerdem musste die Benennung der Standorte und des Medientyps den neuen Gegebenheiten angepasst werden. Und schließlich waren Anpassungen in aDIS für den Betrieb der neuen Buchförderanlage nötig (s. Artikel „Bücher unterwegs").
60.000 Bände des Präsenzbestandes standen bei Eröffnung des Erweiterungsbaus im Gebäude. Das Projekt ging und geht aber weiter: Im Laufe des Jahres werden weitere 90.000 Bände in den Erweiterungsbau gestellt, sofort anschließend erfolgt die Umarbeitung weiterer Bände, die für den Wiedereinzug in das in rund fünf bis sieben Jahren renovierte Bestandsgebäude vorgesehen sind. Durch den tatkräftigen Einsatz aller Projektpartner, allen voran dem BSZ, der Abteilungsleitung Leserservice sowie den beteiligten Kolleginnen und Kollegen von Leihstelle, Medienbearbeitung, IT-Abteilung, Magazin und den zahlreichen Stammund Hilfskräften, die seit Monaten etikettieren und umsortieren, konnte dieses Mammutprojekt in nur 9 Monaten zu einem wichtigen Zwischenziel gebracht werden. Ein großer Dank gilt auch den beteiligten Firmen aStec, Pleuser, Mapongo und Paul v. Maur.

\section{Jörg Oberfell / Birgit Oberhausen / Ralf Pauls} Fotos: Rafael Glatzel

\section{Fazit}

Es ist geschafft! Die Zeit von der Idee im Oktober 2019 bis zur Fertigstellung der ersten zehn Jahrgänge im Juli 2020 war knapp. Die ersten 158.000 Bände aus dem Numerus currens und 